\title{
基于铁催化的 $\mathbf{C}-\mathbf{H}$ 键活化构建 $\mathbf{C}-\mathbf{C}$ 键 的研究进展
}

\author{
牛坡, 温庭斌*
}

厦门大学化学化工学院化学系, 厦门 361005

*通讯作者, E-mail: chwtb@xmu.edu.cn

收稿日期: 2011-01-01; 接受日期: 2011-03-18 doi: 10.1360/032011-1

\begin{abstract}
摘要过渡金属催化的 $\mathrm{C}-\mathrm{H}$ 键活化及在此基础上的 $\mathrm{C}-\mathrm{C}$ 键形成的反应因其高原子经济性和 高效的合成效率而备受人们的关注。铁元素具有含量丰富、廉价、易得、环境友好等优点, 在 催化反应中得到了越来越广泛的应用. 近几年来, 人们关于 $\mathrm{Fe}$ 催化的 $\mathrm{C}-\mathrm{H}$ 键活化构建 $\mathrm{C}-\mathrm{C}$ 键 反应的研究也取得了一定的进展. 本文对铁催化的 $\mathrm{C}-\mathrm{H}$ 键活化构建 $\mathrm{C}-\mathrm{C}$ 键的最新研究进展作 了综述, 并且按照铁催化剂的不同价态进行了分类归纳, 也对催化机理进行了阐述与总结.

关键词

铁

催化

$\mathrm{C}-\mathrm{H}$ 键活化

C-C 键的构建
\end{abstract}

\section{1 引言}

$\mathrm{C}-\mathrm{C}$ 键的构建是有机合成领域的重要研究内容 之一 ${ }^{[1]}$, 发展高效、高选择性的 $\mathrm{C}-\mathrm{C}$ 键形成的新反应、 新途径将会大大提高目标产物的合成效率, 进而对现 代有机合成化学的发展和应用产生积极的影响, 具有 重大的理论意义和应用价值 ${ }^{[2]}$. 过渡金属催化已成为 构建 $\mathrm{C}-\mathrm{C}$ 键的重要策略之一. 在催化形成 $\mathrm{C}-\mathrm{C}$ 键的过 程中, 经常会涉及到如卤素等活性官能团的使用 ${ }^{[3]}$.

这其中的关键步骤如图 1 所示, 首先低价态的金 属经过氧化加成插入到 $C-X(X$ 为卤素等)键之间形成 $\mathrm{C}-\mathrm{M}$ 键, 然后烯烃、炔烃、一氧化碳等小分子化合物 插入到 $\mathrm{C}-\mathrm{M}$ 键之间形成新的 $\mathrm{C}-\mathrm{C}$ 键, 再经过 $\beta-\mathrm{H}$ 消

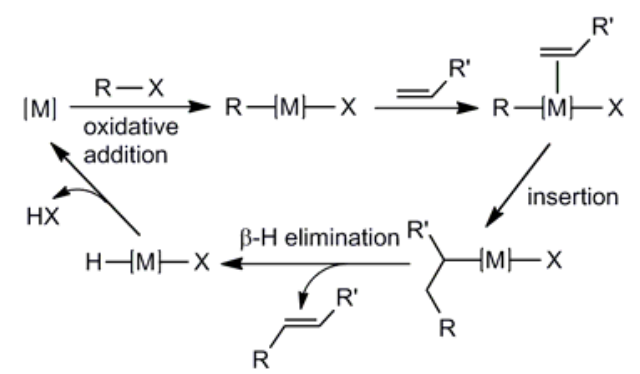

图 1 传统的过渡金属催化的 $\mathrm{C}-\mathrm{C}$ 键偶联
除生成新的有机产物, 并通过消除 HX 再生金属催化 剂(类 Heck 反应机理).

以 $\mathrm{C}-\mathrm{H}$ 键活化为基础的有机转换使得我们在设 计合成反应路线时可以象考虑 $\mathrm{C}-\mathrm{X}$ ( $\mathrm{X}$ 为卤素) 一样 来考虑 $\mathrm{C}-\mathrm{H}$ 键, 从而可能减少合成步骤、提高原子 利用率 ${ }^{[4]}$, 可望发展高效、经济的合成策略, 为化学 反应合成路线的设计提供新的思路 ${ }^{[5]}$. 因此过渡金属 促进的 $\mathrm{C}-\mathrm{H}$ 键活化以及在此基础上的 C-C 键构建的 研究引起了化学家们的广泛关注 ${ }^{[6]}$.

C-H 键活化研究的思路是寻找合适的催化剂, 通过选择性地活化 $\mathrm{C}-\mathrm{H}$ 键, 并进一步实现在被活化 的 C-H 键位置的官能团化或促进新的化学键的形成. 首例过渡金属促进的 C-H 活化报道于 1963 年, Kleiman 和 Dubeck 实现了 $\mathrm{Cp}_{2} \mathrm{Ni}$ 对偶氮苯计量化的 $\mathrm{C}-\mathrm{H}$ 键断裂, 生成环金属化的产物 ${ }^{[7]}$. 经过半个世纪 的发展, C-H 键活化研究已取得了巨大的进步, 无论 是在计量化研究方面还是催化反应的研究. 现在几 乎所有的过渡金属都已有相关的计量化 $\mathrm{C}-\mathrm{H}$ 键活化 的报道 ${ }^{[8]}$, 包括 $\mathrm{Ru}^{[9]} 、 \mathrm{Ir}^{[10]} 、 \mathrm{Os}^{[11]} 、 \mathrm{Rh}^{[12]} 、 \mathrm{Pd}^{[13]}$ 等贵 金属, 以及 $\mathrm{Cu}^{[14]} 、 \mathrm{Fe}^{[15]}$ 等廉价易得的金属, 还有不少 稀土金属促成的 $\mathrm{C}-\mathrm{H}$ 键活化的报道 ${ }^{[16]}$. 和计量化研 
究相比, 基于 C-H 键活化从而进一步实现有机催化 反应的报道虽然相对较少, 但是 经过科学家们不解 的努力, 该领域在最近十年里取得了突破性的进展, 特别是在 $\mathrm{Ru}^{[17]} 、 \mathrm{Rh}^{[18]} 、 \mathrm{Pd}^{[19]}$ 等稀有贵金属催化的 $\mathrm{C}-\mathrm{H}$ 键活化研究上, 一系列引人关注的成果不断涌现.

另一方面, 发展新型、成本低廉、环境友好的催 化剂一直是化学家们关注的研究方向. 铁作为地球 中含量最丰富的金属元素, 具有无毒、环境友好等优 点, 是理想的绿色化学催化剂, 在催化反应中也得到 了越来越广泛的应用 ${ }^{[20]}$. 和 $\mathrm{Ru} 、 \mathrm{Rh} 、 \mathrm{Pd}$ 等贵金属相 比, 铁催化的 C-H 键活化的研究虽然相对较少, 近 年来, 这方面的研究也有了很大的发展, 已有多篇相 关方面的综述报道 ${ }^{[21]}$. 本文对近几年铁催化的 $\mathrm{C}-\mathrm{H}$ 键活化构建 $\mathrm{C}-\mathrm{C}$ 键的研究进展进行综述, 并且按照 铁催化剂的不同价态进行了分类归纳.

\section{2 铁催化的 C-H 键活化构建 $\mathbf{C}-\mathrm{C}$ 键}

\section{$2.1 \mathrm{Fe}^{0}$ 催化剂}

早在 1987 年 Jones 课题组 ${ }^{[22]}$ 就报道了 $\mathrm{Fe}^{0}$ 形式的 $\mathrm{Fe}\left(\mathrm{PMe}_{3}\right)_{2}\left(\mathrm{CNBu}^{t}\right)_{3}$ 可以催化异氧类化合物与苯、甲 苯、苯乙烯等芳烃类化合物在紫外光照条件下发生加 成反应生成亚胺类化合物 2(图 2).

此反应的可能机理如图 3 所示, 首先光照促使 $\mathrm{Fe}\left(\mathrm{PMe}_{3}\right)_{2}\left(\mathrm{CNBu}^{t}\right)_{3}$ 的异氧配体解离, 产生四配位 $\mathrm{Fe}^{0}$ 中间体 $\mathbf{4}$ 即为此反应中起催化作用的物种, 低价态富 电子的铁配合物 $\mathbf{4}$ 和苯发生氧化加成, 然后一个配位 的异氭插入到 $\mathrm{Fe}-\mathrm{Ph}$ 键之间, 紧接着另一分子的异氧 再配位到 $\mathrm{Fe}$ 上生成中间体 7 , 最后经过还原消除得到 亚胺类化合物 2 , 同时再生催化剂参与下一个催化循 环.

2008 年, 李志平课题组 ${ }^{[23]}$ 以 $\mathrm{Fe}_{2}(\mathrm{CO})_{9}$ 为催化剂, 在 ${ }^{t} \mathrm{BuOO}{ }^{t} \mathrm{Bu}$ 做氧化剂的条件下, 通过活化醚, 硫醚, 或者胺类底物中杂原子邻位的 $\mathrm{C}-\mathrm{H}$ 键, 实现其与二 羰基化合物的偶联(图 4).

如图 5 所示, 无论是环状醚类化合物 8a-8c, 链

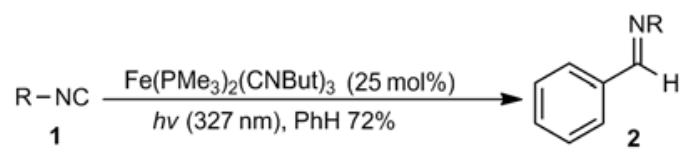

图 2 苯与氧类化合物的偶联 ${ }^{[22]}$

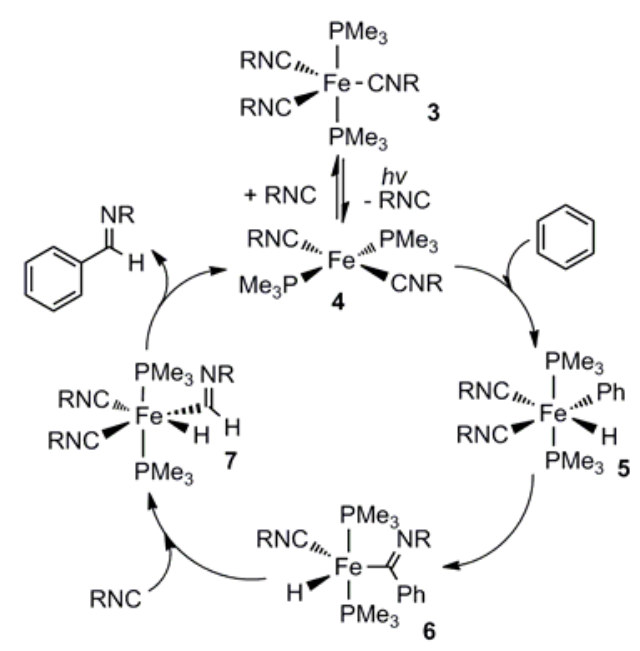

图 3 苯与氰类化合物的偶联反应机理 ${ }^{[22]}$

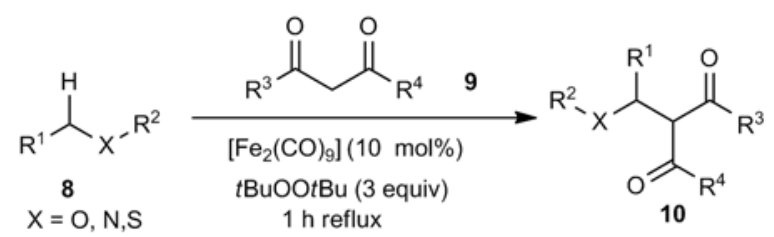

图 4 铁催化的杂原子邻位 $\mathrm{C}-\mathrm{H}$ 键活化及与二羰基化合物 的偶联 ${ }^{[23]}$

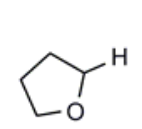

$8 \mathbf{a}$<smiles>COCc1ccccc1</smiles>

8 e<smiles>C1CCCC1</smiles>

$8 b$<smiles>c1ccc(COCc2ccccc2)cc1</smiles>

$8 \mathrm{~g}$<smiles>CCOC(C)C</smiles>

$8 d$<smiles>CN(C)Pc1ccccc1</smiles>

8 h<smiles>O=C(CC(=O)c1ccccc1)c1ccccc1</smiles>

9a<smiles>CC(=O)CC(=O)c1ccccc1</smiles>

$9 b$<smiles>O=C(CC(=O)Nc1ccccc1)Nc1ccccc1</smiles>

9c<smiles>CCOC(=O)CC(C)=O</smiles>

图 5 代表性的含杂原子化合物与二羰基类化合物 ${ }^{[23]}$

状醚类化合物 8d-8f, 硫醚类化合物 8g 亦或者是胺类 化合物 $8 \mathrm{~h}$ 在铁和过氧化物催化条件下, 都可以通过 活化杂原子邻位的 C-H 键与二羰基化合物(如 9a-9f 等) 反应得到 $\mathrm{C}-\mathrm{C}$ 键偶联的产物 10. 各种类型底物的 拓展发展了合成此类含杂原子化合物的方法, 也为 
日后此类反应在天然产物、药物等合成中的可能应用 打下了基础.

但是当胺类化合物如 $\mathbf{8 h}$ 在 $\mathrm{Fe}_{2}(\mathrm{CO})_{9}$ 和 $t$ $\mathrm{BuOOH}(\mathrm{TBHP})$ 催化下与二羰基化合物反应时, 却选 择性的得到了 $\mathrm{C}-\mathrm{N}$ 键切断的亚甲基桥联的四羰基化 合物 11(图 6) ${ }^{[24]}$. 而且不仅是 $N, N$-二甲基苯胺, 其他 的叔胺(如图 7 所示的 $(\mathbf{8 h}-\mathbf{a}-\mathbf{8 h}-\mathbf{d}))$ 也可以提供反应 桥连所需的亚甲基, 与二羰基化合物发生相同的反 应。

研究发现, 如果 $\beta$-二羰基化合物 9 与过量的 $N, N$ 二甲基苯胺 $8 \mathrm{~h}$ 在标准条件下反应生成的是二羰基产 物 $\mathbf{1 0}$ 和亚甲基桥联的四羰基化合物 $\mathbf{1 1}$ 的混合物(图 8), 而二羰基产物 10 在 $\mathrm{Fe}_{2}(\mathrm{CO})_{9}$ 和 ${ }^{t} \mathrm{BuOOH}$ 催化下可 继续与 $\beta$-二羰基化合物 9 反应生成四羰基化合物 $\mathbf{1 1}$, 如果所用的 $\beta$-二羰基化合物不同, 则可得到相应的交 叉偶联的四羰基产物.

因此该反应可能的机理是(图 8): 二羰基化合物 和甲基胺类化合物首先在 $\mathrm{Fe}_{2}(\mathrm{CO})_{9}$ 和 ${ }^{t} \mathrm{BuOOH}$ 催化 下发生偶联反应生成化合物 $\mathbf{1 0}$, 然后在体系中的 $\mathrm{Fe}$ 路易斯酸催化下发生 C-N 键断裂, 并且与另外一分 子 $\beta$-二羰基化合物通过 $\mathrm{S}_{N} 2$ 反应抑或是 Cope 消除和 Michael 加成得到四羰基产物 11.

\section{$2.2 \mathrm{Fe}^{\mathrm{II}}$ 催化剂}

2003 年, McGill 大学的李朝军教授 ${ }^{[25]}$ 首先提出 了交叉脱氢偶联 $(\mathrm{CDC})$ 的概念, 即直接利用不同反应 底物中的 C-H 键在氧化条件下进行交叉偶联反应形 成 $\mathrm{C}-\mathrm{C}$ 键.

2007 年, 李志平课题组 ${ }^{[26]}$ 首次报道了铁催化的

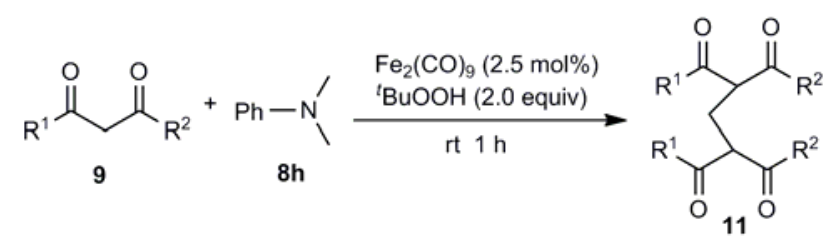

图 6 铁催化的 $N$-甲基苯胺的选择性氧化及其与二羰基化 合物的偶联 ${ }^{[24]}$

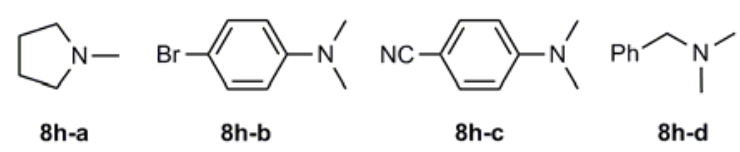

图 7 各种类型的叔胺底物 ${ }^{[24]}$

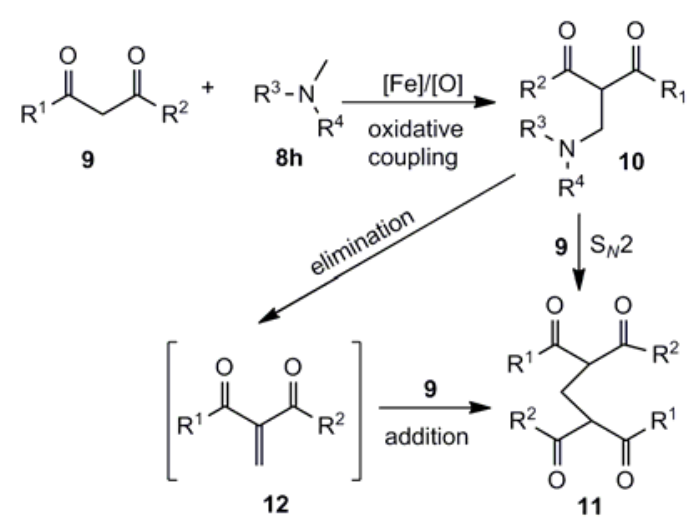

图 8 二羰基化合物与叔胺偶联的可能反应机理 ${ }^{[24]}$

$\mathrm{CDC}$ 反应, 成功实现了铁催化脱氢构建 $\mathrm{C}-\mathrm{C}$ 键. 苄 基类化合物的 $\mathrm{C}-\mathrm{H}$ 键经过 $\mathrm{FeCl}_{2}$ 催化活化, 在室温条 件下即可与二羰基类化合物反应得到 $\mathrm{C}-\mathrm{C}$ 键的偶联 产物(图 9). 研究结果表明, 无论是带有吸电子基团 的芐基 13b 还是供电子基团的芐基 $13 \mathrm{c}$ (图 10), 芐基 的 $\mathrm{C}-\mathrm{H}$ 键都可以经过氧化活化与各种二羰基类化合 物(14a-14e) 实现高效高选择性的 C-C 键的构建.

反应的可能机理如图 11 所示, 首先是过氧化叔 丁醇将 $\mathrm{Fe}$ 吕氧化成 $\mathrm{Fe}{ }^{\mathrm{III}}$ 配合物 16 , 同时产生叔丁氧 自由基 17, 而苄基类化合物与二羰基类化合物在 16 和 17 的作用下, 生成二羰基与 $\mathrm{Fe}^{\mathrm{III}}$ 配位的中间体 $\mathbf{1 8}$, 同时受新产生的茮基自由基 19 的进攻, 得到最终产 物 15 和再生的 $\mathrm{Fe}^{\mathrm{II}}$ 催化剂.

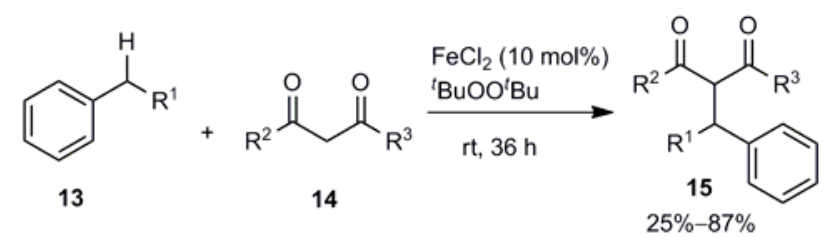

图 9 茮基类化合物的 $\mathrm{C}-\mathrm{H}$ 键活化 ${ }^{[26]}$
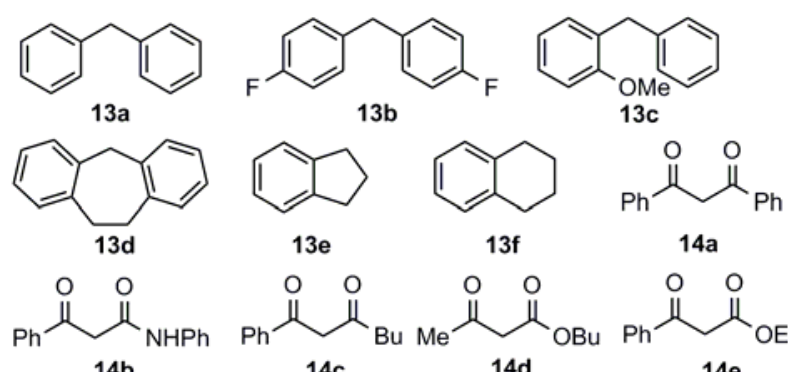
${ }_{\mathrm{Ph}}^{\mathrm{O}} \mathrm{N}_{\mathrm{OEt}}^{\mathrm{O}}$

图 10 代表性的苄基化合物与二羰基化合物 ${ }^{[26]}$ 


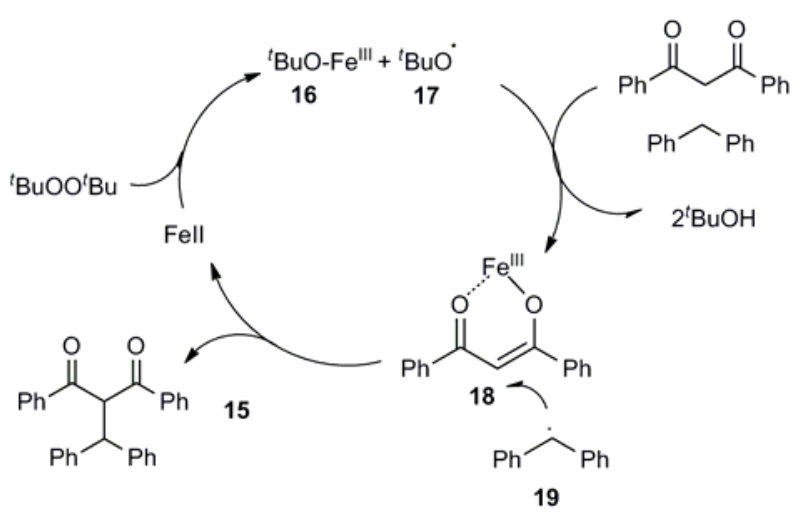

图 11 茮基类化合物与二羰基化合物的偶联反应机理 ${ }^{[26]}$

2007 年, 李朝军课题组 ${ }^{[27]}$ 发现环状烷烃或者链 状烷烃亦可以在 $\mathrm{FeCl}_{2} \cdot 4 \mathrm{H}_{2} \mathrm{O}$ 和过氧化物 ${ }^{t} \mathrm{BuOO}^{t} \mathrm{Bu}$ 的催化下选择性活化 $\mathrm{C}-\mathrm{H}$ 键并和二羰基化合物 21 发 生偶联反应，从而实现 $\mathrm{sp}^{3}-\mathrm{sp}^{3} \mathrm{C}-\mathrm{C}$ 键的偶联(图 12).

以上反应均是通过活化反应体系中一种底物的 $\mathrm{C}-\mathrm{H}$ 键从而实现 $\mathrm{C}-\mathrm{C}$ 健的构建. 2009年, 施章杰课题 组 ${ }^{[28]}$ 则报道了 $\mathrm{FeCl}_{2}$ 和 $\mathrm{DDQ}$ 催化活化茮基的 $\mathrm{C}\left(\mathrm{sp}^{3}\right)-\mathrm{H}$ 键和富电子芳烃的 $\mathrm{C}\left(\mathrm{sp}^{2}\right)-\mathrm{H}$ 键, 从而实现 通过活化两种不同的 $\mathrm{C}-\mathrm{H}$ 键基础上的 $\mathrm{C}\left(\mathrm{sp}^{3}\right)-\mathrm{C}\left(\mathrm{sp}^{2}\right)$ 键的偶联(图 13), 并将此类反应称为交叉脱氢芳基化 反应(CDA 反应).

如图 13 所示, 既含 $\mathrm{OMe}$ 供电子基又含 $\mathrm{CO}_{2} \mathrm{Me}$ 吸电子基的 2-甲氧基苯甲酸甲酯 23 在 $\mathrm{FeCl}_{2}$ 和 $\mathrm{DDQ}$ 催化氧化下和二苯甲烷 24a 发生反应, 生成甲氧基对 位和二苯甲烷亚甲基偶联的产物 25, 2-甲氧基苯甲酸 甲酯并未与二苯甲烷的苯基发生反应，而且生成的 产物 25 也未继续进行后续转化, 这些都充分说明了

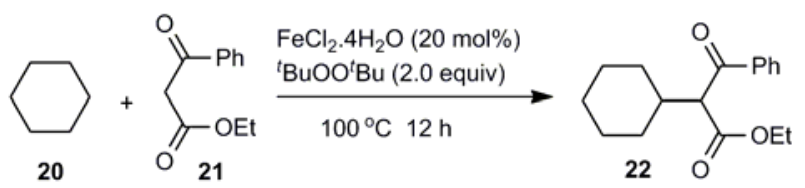

图 12 环己烷与二羰基化合物的偶联 ${ }^{[27]}$

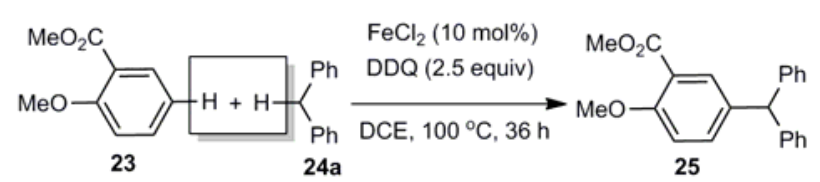

图 13 2-甲氧基苯甲酸甲酯与二苯甲烷的偶联 ${ }^{[28]}$
此反应具有高度的化学选择性. 研究发现电子效应 对反应有较大影响, 例如当苯甲醚的 2 位为醛基、卤 素等吸电子基团时, 反应都能得到较好收率的甲氧 基对位和茮基偶联的产物.

当苯甲醚苯基上没有取代基时，与茮基化合物 24a 反应却得到了甲氧基邻位取代的偶联产物 27 和 甲氧基邻、对位都被取代的产物 28 的混合物(图 14).

当 1,2,3-苯三甲醚 $\mathbf{2 9}$ 与苠基化合物 $24 \mathbf{a}$ 反应时 则得到了 4,6 位被二苯甲烷取代的偶联化合物 30 (图 15).

当苄基底物中苯环的 2 位含有甲基等基团或者 苯环上含有吸电子基团时反应的产率就会降低，电 子效应的影响表明该反应为付-克类型的反应. 结合 所做的気代实验, 推断此反应的可能机理如图 16 所 示: 首先 $\mathrm{Fe}^{\mathrm{II}}$ 被 $\mathrm{DDQ}$ 氧化为 $\mathrm{Fe}^{\mathrm{III}}$ 与 $\mathrm{DDQ}$ 的配位化合 物 31, 并且产生二苯甲基自由基, 二苯甲基自由基 和中间体 31 发生单电子转移, 生成二苯甲基阳离子 和中间体 32, 阳离子与 2-甲氧基苯甲酸甲酯反应生 成中间体 33 , 中间体 32 再夺取 33 的一个质子, 得到 最终产物 25 并再生 $\mathrm{Fe}^{\mathrm{II}}$ 催化剂.

该课题组进一步的研究发现苠基化合物 $\mathbf{2 4}$ 亦可 以与苯乙烯类化合物 $\mathbf{3 5}$ 在 $\mathrm{FeCl}_{2}$ 和 DTBP 催化下发 生类似于 Heck 形式的偶联反应 ${ }^{[29]}$. 在温和的条件下, 多种苠基类底物都可以经过 $\mathrm{C}-\mathrm{H}$ 活化与苯乙烯类化 合物高效高选择性的构建 $\mathrm{C}-\mathrm{C}$ 键(图 17).

同时，在考察反应对如图 18 所示的底物的选择 性时发现, 无论是苠基上带有吸电子基团如 24b, 还 是给电子基团如 $\mathbf{2 4 c}$, 亦或是苯乙烯的苯环上带有给

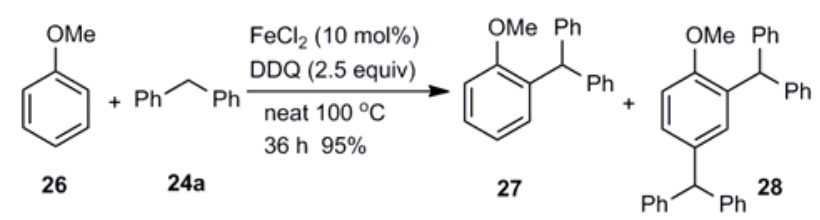

图 14 苯甲醚与二苯甲烷的偶联 ${ }^{[28]}$<smiles>COc1cccc(OC)c1OC</smiles>

29

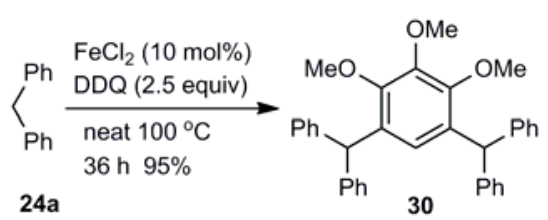

24a
30
图 15 苯三甲醚与二苯甲烷的偶联 ${ }^{[28]}$ 


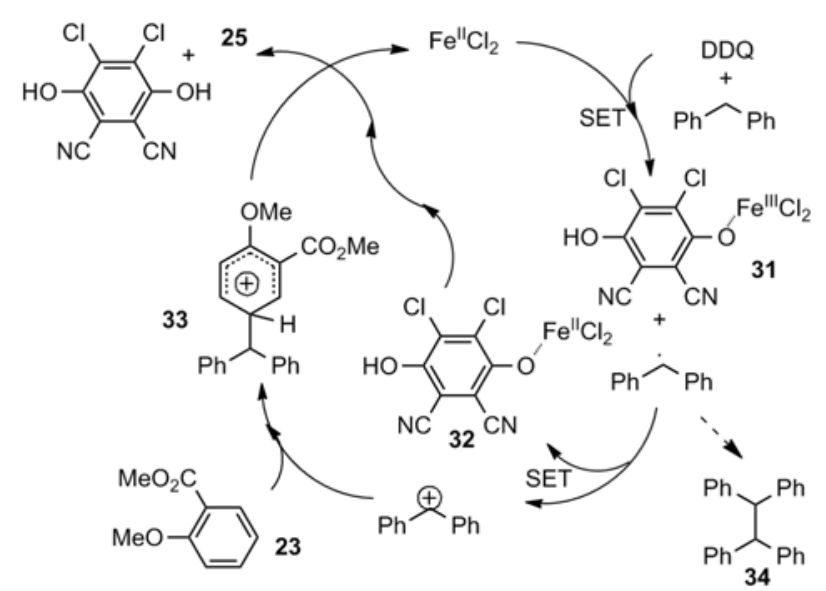

图 16 茮基类化合物的 CDA 反应机理 ${ }^{[28]}$

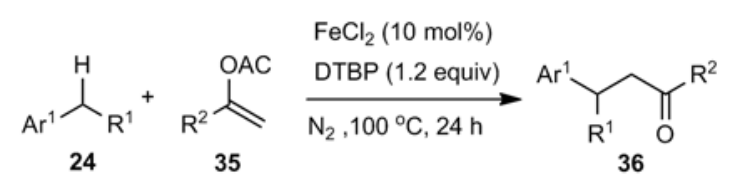

图 17 茮基化合物与烯的偶联 ${ }^{[29]}$<smiles>COc1ccc(Cc2ccccc2)cc1</smiles>

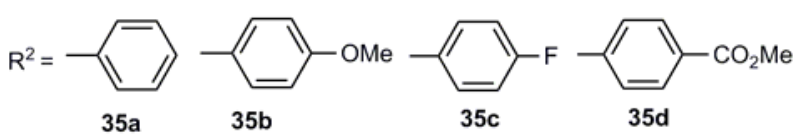

图 18 代表性的茮基类化合物及烯烃的取代基 $\mathrm{R}^{2 \text { [29] }}$

电子基团如 35a、35b, 或者是苯环上带有吸电子基团 如 35c、35d, 在标准条件下, 二者都可以反应得到较 好收率的酮类产物 36.

联芳基类化合物是存在于大自然中的重要生物 活性物质，此类化合物的合成方法多数是用 $\mathrm{Ru} 、 \mathrm{Rh}$ 、 Pd 等过渡金属催化的偶联反应 ${ }^{[30]}$. 2009 年, Charette 课题组 ${ }^{[31}$ 报道了利用 $\mathrm{Fe}(\mathrm{OAc})_{2}$ 催化活化苯基的 $\mathrm{C}-\mathrm{H}$ 键与碘苯合成联芳基类化合物的反应. 如图 19 所示, 在 $5 \mathrm{~mol} \% \mathrm{Fe}(\mathrm{OAc})_{2} 、 10 \mathrm{~mol} \%$ 4,7-二苯基-1,10-菲咯 啉及 2.0 equiv $\mathrm{KO} t$-Bu 的催化体系下, 碘苯 $\mathbf{3 7}$ 和苯 $\mathbf{3 8}$ 温和条件下即可以反应生成联苯类化合物 39. 而对溴 甲苯在此反应条件下反应效果较差, 对氯甲苯则几乎 不发生类似反应. 对于反应可能的机理, 可认为首先 是碘苯在 $\mathrm{Fe}^{\mathrm{II}}$ 催化下通过单电子转移氧化产生苯基 自由基, 而 $\mathrm{Fe}^{\mathrm{II}}$ 则被氧化生成 $\mathrm{Fe}^{\mathrm{III}}$ 中间体, 苯基自由

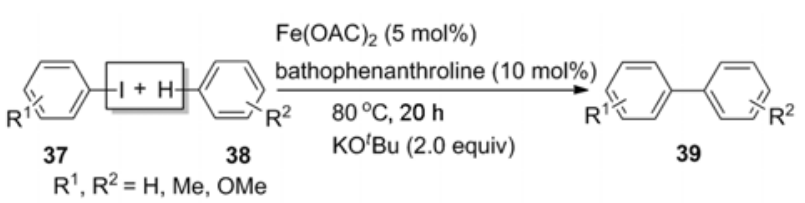

图 19 碘苯与芳烃的偶联 ${ }^{[31]}$

基进攻芳基并且在 $\mathrm{Fe}^{\mathrm{III}}$ 中间体和叔丁醇钾作用下脱 去一分子 $\mathrm{HI}$, 得到联苯类化合物.

含氮化合物广泛存在于药物、生物体内, 是一类 重要的生物活性和药物活性中间体, 有关它的合成 以及偶联反应一直是人们研究的热点. 2009 年, Vogel 课题组 ${ }^{[32]}$ 研究发现 $\mathrm{FeCl}_{2}$ 可以选择性催化活化叔胺类 化合物 $N$-甲基的 $\mathrm{C}-\mathrm{H}$ 键，进而与末端炔烃发生反应， 实现 $\mathrm{C}\left(\mathrm{sp}^{3}\right)-\mathrm{C}(\mathrm{sp})$ 的偶联(图 20). 末端炔烃类化合物 41 在标准反应条件下与叔胺类化合物 40 的 $N$ - Me 进 行了偶联, 而未与苯基反应, 说明了此反应具有较好 的化学选择性. 此反应在空气条件下可以得到较好 的收率, 而在 $1 \mathrm{~atm} \mathrm{O}_{2}$ 条件下反应的效率却大大降 低. 因此该反应中 $N-\mathrm{Me}$ 上自由基 $\mathrm{R}_{2} \mathrm{NCH}_{2} \mathrm{R}^{\prime}$ 的产生 可能是反应的关键步骤, 后者即和 $\mathrm{O}_{2}$ 能够捕捉并且 猝灭此自由基有关. 而且, 当反应体系中有水存在 时, 反应时间增长, 并会有难以分离的副产物 43 产 生(图 21). 因此, 该反应必须在无水条件下进行.

\section{$2.3 \mathrm{Fe}^{\mathrm{III}}$ 催化剂}

2008 年, Nakamura 课题组 ${ }^{[33,34]}$ 首次报道了芳基 锌化合物(通过格氏试剂与 $\mathrm{ZnCl}_{2}$ 反应生成)与苯并喹 啉类化合物在铁的催化下的偶联反应. 如图 22 所示, 反应以 $10 \mathrm{~mol} \%$ 的 $\mathrm{Fe}(\mathrm{acac})_{3}$ 为催化剂, $10 \mathrm{~mol} \%$ 的

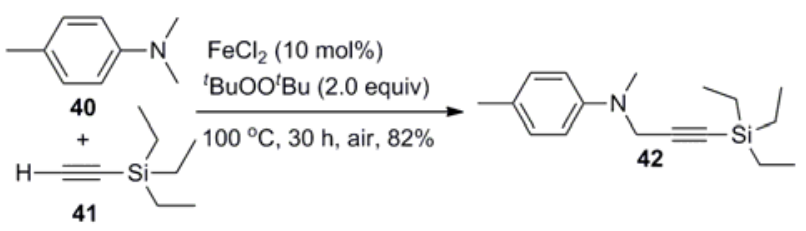

图 $20 N$-甲基的选择性 C-H 键活化 ${ }^{[32]}$

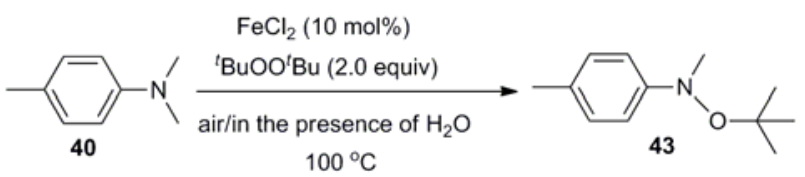

图 21 水对 $N$-甲基选择性活化 $\mathrm{C}-\mathrm{H}$ 键的反应影响 ${ }^{[32]}$ 


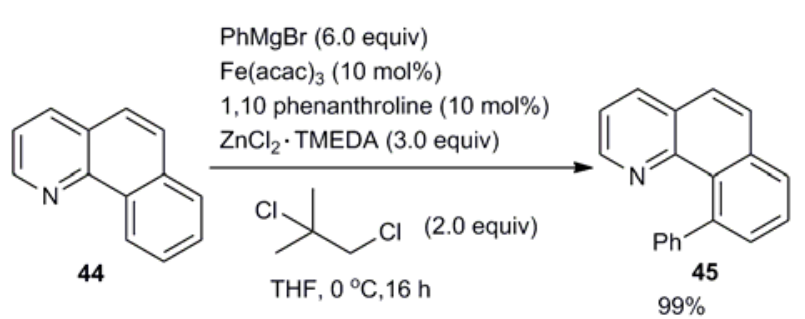

图 22 苯并喹啉与芳基锌试剂的偶联 ${ }^{[33]}$

1,10-菲咯啉为配体, 在 THF 溶剂中加入苯并哇啉 44 、 6 equiv $\mathrm{PhMgBr} 、 3$ equiv $\mathrm{ZnCl}_{2}$ TMEDA、 2 equiv 1,2二氯-2-甲基丙烷, $0{ }^{\circ} \mathrm{C}$ 条件下反应 $16 \mathrm{~h}$, 在喹啉环上 氮杂原子的化学选择性控制下, 活化苯并喹啉 10 位的 C-H 键, 几乎定量地得到偶联产物 45. 而且在标准条 件下, 无论是苯并喹啉 4 位上还是格氏试剂苯环上带 有吸电子基团或者给电子基团, 反应都能很好的进行 以得到高化学选择性和较好收率的偶联产物. 但是随 着芳基吡啶苯环上 2,3 位有取代基时, 偶联反应的产 率会逐渐降低, 而且当 2 位有较大位阻的取代基时, 偶联反应甚至无法进行, 说明位阻效应对该反应有较 大影响.

如果用 2-苯基嘧啶 46 与苯基锌试剂反应(图 23), 则可生成苯基取代基 2 位被取代的化合物 47 和苯基 2,6 位都被取代的化合物 $\mathbf{4 8}$ 的混合物.

在上述反应的基础上, 进一步研究发现在该催 化体系中, 芳基亚胺类化合物 49 也可以通过亚胺 $\mathrm{N}$ 原子控制化学选择性活化芳基 2 位的 $\mathrm{C}-\mathrm{H}$ 键并且与 芳基锌试剂发生偶联反应(图 24), 偶联产物再水解得 到 2-苯基苯乙酮类化合物 $\mathbf{5 0}^{[35]}$. 并且发现芳基亚胺 类化合物的苯环 3,4 位含有卤素、对甲苯磺酰基、三 氟甲磺酰基等易离去基团时, 反应仍然能够很好的 进行, 这也进一步说明此反应体系具有高度的化学 选择性, 但该反应缺点是需要消耗大量的昂贵氧化

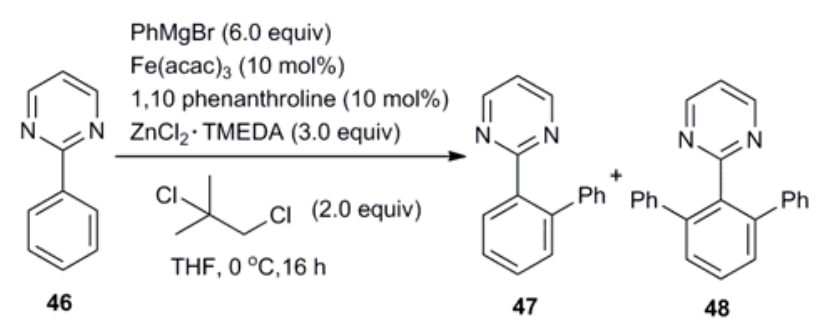

图 23 2-苯基嘧啶与芳基锌试剂的偶联 ${ }^{[33]}$

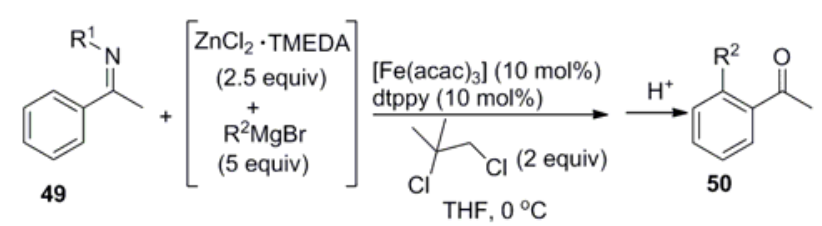

图 24 亚胺与有机锌试剂的偶联 ${ }^{[35]}$

剂 1,2-二氯-2-甲基丙烷.

Yoshikai 等 ${ }^{[36]}$ 也尝试了利用 $\mathrm{O}_{2}$ 替代二氯烷烃作 为反应的氧化剂, $\mathrm{FeCl}_{3}$ 替代 $\mathrm{Fe}(\mathrm{acac})_{3}$ 作为反应的催 化剂, 以改善反应的催化剂体系(图 25). 研究发现, 当苯亚胺化合物苯环上无取代基时，反应的产率达 到 80\%. 但是当用此反应体系催化活化芳基吡啶类 化合物的 $\mathrm{C}-\mathrm{H}$ 键构建新的 $\mathrm{C}-\mathrm{C}$ 键时, 反应的效率较 前者却大大降低，而且反应需要消耗比前者更多的 芳基锌试剂.

而当 Yoshikai 等研究对甲碘苯和 $\mathrm{Ph}_{2} \mathrm{Zn}$ 在以

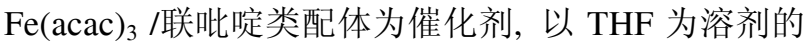
反应体系中反应时, 并未得到碘苯和苯基偶联的产 物, 而是意外地得到了呋喃环上的 2 位和苯基偶联的 产物(图 26), 而且是 $\mathrm{Ph}_{2} \mathrm{Zn}$ 的苯基而不是碘苯和 $\mathrm{THF}$ 偶联 ${ }^{[33,35,36]}$. 对于反应的可能机理,可认为: 碘苯首 先在铁催化剂作用下产生芳基自由基，芳基自由基 再夺取 THF 的 2 位上的氢, 从而发生 THF 自由基和 $\mathrm{Ph}_{2} \mathrm{Zn}$ 苯基的偶联.

该反应的结果激发了研究类似结构的脂肪胺通 过铁催化实现其 $\alpha$ 位的 $\mathrm{sp}^{3} \mathrm{C}-\mathrm{H}$ 键的活化并和锌试剂 或者格氏试剂 $\mathrm{RM}$ 偶联的兴趣 ${ }^{[37]}$. 因此，以 $\mathrm{N}$ 上带 2碘代苄基的脂肪胺 ( $N$-IBn) 为底物 (如吡咯类衍生物 54), 在 $2.5 \mathrm{~mol} \% \mathrm{Fe}(\mathrm{acac})_{3}$ 催化下和 $\mathrm{PhMgBr}$ 或 $\mathrm{Ph}_{2} \mathrm{Zn}$

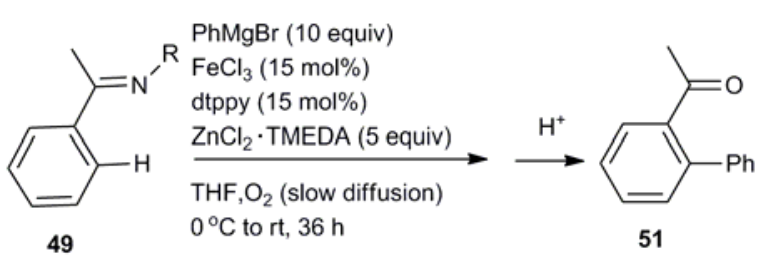

图 $25 \mathrm{FeCl}_{3}$ 催化的亚胺的选择性 C-H 键活化 ${ }^{[36]}$

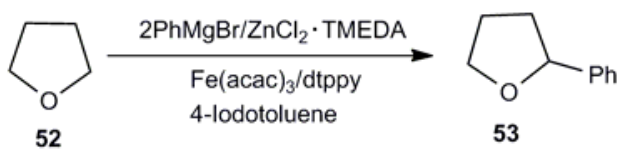

图 26 四氢呋喃与苯基锌试剂的偶联 ${ }^{[37]}$ 
反应得到了 $80 \%$ 的目的产物 $\mathbf{5 5}$ (图 27). 由于 THF 会 参与反应, 因此用 $\mathrm{Et}_{2} \mathrm{O}$ 作为此类反应的溶剂, 反应 条件更加温和.

不仅吡咯类衍生物, 六元环、七元环等环状胺, 甚至是非环的脂肪胺等在此反应体系中都可以得到 较好收率的产物. 对于各种类型的格氏试剂, 即使如 氯苯基、丙烯基格氏试剂亦可以与吡咯类衍生物 $\mathbf{5 4}$ 反应而得到较好收率的产物. 对于底物 $\mathbf{5 6}$, 由于 $\mathrm{N}$ 邻 位的甲基和亚甲基都可能被活化，因此与格氏试剂 反应可生成 $\mathbf{5 7}$ 和 $\mathbf{5 8}$ 两种产物(图 28).

这个反应结果也间接证实了对反应机理的推断. 结合进行的氝代实验, 认为反应可能的机理如图 29 所示: 含 2-碘代芐基的胺类化合物 $\mathbf{5 4}$ 在铁的催化下 产生自由基中间体 $\mathbf{5 9}$, 然后 $\mathrm{N}$ 原子邻位的 $\mathrm{C}$ 上的氢 经过 1,5 氢转移, 生成自由基中间体 $\mathbf{6 0}, \mathbf{6 0}$ 再与由锌

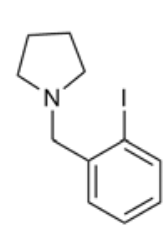

54

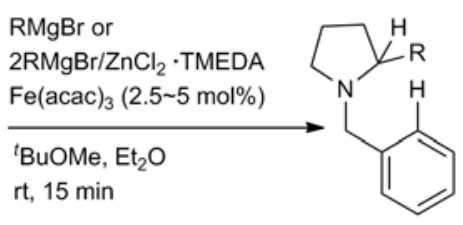

55

图 27 脂肪胺 $\alpha$ 位的 $\mathrm{sp}^{3} \mathrm{C}-\mathrm{H}$ 键活化 ${ }^{[37]}$

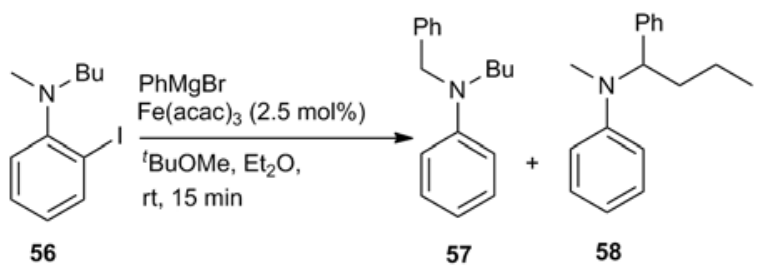

图 282 -碘-( $N$-甲基- $N$-丁基)-苯胺与格氏试剂的偶联 ${ }^{[37]}$
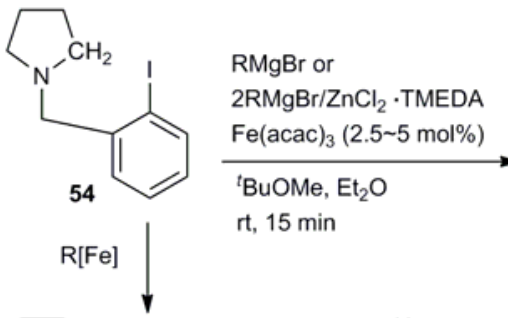

$\mathrm{rt}, 15 \mathrm{~min}$

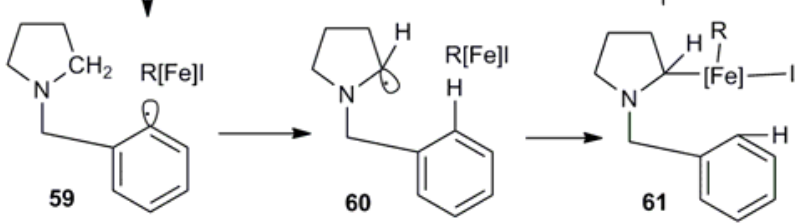

图 29 脂肪胺与锌试剂反应的可能机理 ${ }^{[37]}$
试剂或者格氏试剂和 $\mathrm{Fe}$ 催化剂产生的烃基铁物种反 应，最后经过还原消除得到偶联产物 $\mathbf{5 5}$, 铁催化剂 则进入下一个催化循环. 上述反应共同的缺点是需 要大量的格氏试剂和芳基锌试剂, 而且对底物的要 求较高.

Pd 催化的 Suzuki 偶联反应因为其适用于各类硼 酸与烯基澳、芳基澳的偶联而广泛应用在化学研究和 工业生产中 ${ }^{[38]}$. 但是 $\mathrm{Pd}$ 催化剂价格昂贵, 如能用廉 价金属替代 $\mathrm{Pd}$ 催化偶联反应则更为理想. 2008 年, 余孝其课题组 ${ }^{[39]}$ 报道了温和条件下 $\mathrm{Fe}_{2}\left(\mathrm{SO}_{4}\right)_{3} \cdot 7 \mathrm{H}_{2} \mathrm{O}$ 催化的直接活化芳基 $\mathrm{C}-\mathrm{H}$ 键与芳基硼酸的类 Suzuki 偶联反应(图 30).

该反应体系中, $1,4,7,10$ 四氮杂环十二烷(cyclen) 为配体, $\mathrm{K}_{3} \mathrm{PO}_{4}$ 作为碱, pyrazole 是反应中必需的添加 剂. 反应如果在氮气氛下进行, 则得不到偶联的产 物, 在空气中反应则可以顺利进行, 说明 $\mathrm{O}_{2}$ 是反应 所必需的氧化剂. 在拓展底物(如图 31)时发现苯基硼 酸 3,4 位有取代基时如 62a、62b, 反应可以较好的进 行，而当 2 位有取代基如 $62 \mathrm{c}$ 时，则几乎得不到偶联 的目的产物，从而说明了位阻效应对该反应的影响. 而当苯硓酸上含有羧酸取代基如 62d 时, 也未得到预 想的偶联产物, 说明了反应对于酸的敏感性. 对于底 物 63, 当苯环上含有吸电子基团时, 反应的产率会 有所提高, 说明电子效应也会对反应产生一定的影 响.

前文提到 Nakamura 课题组主要研究了通过活化

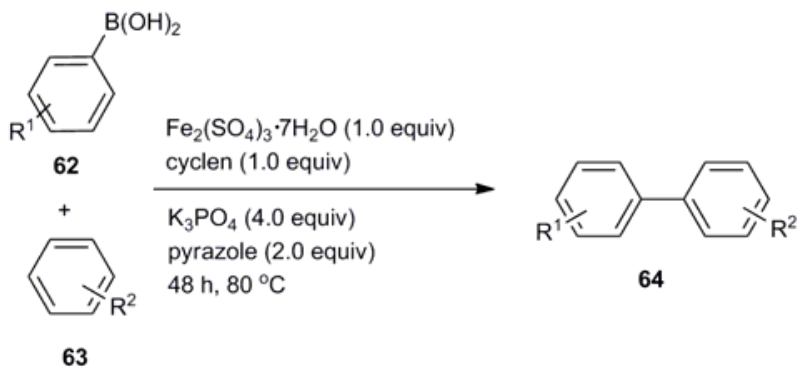

图 30 苯嗍酸与芳烃的偶联 ${ }^{[39]}$

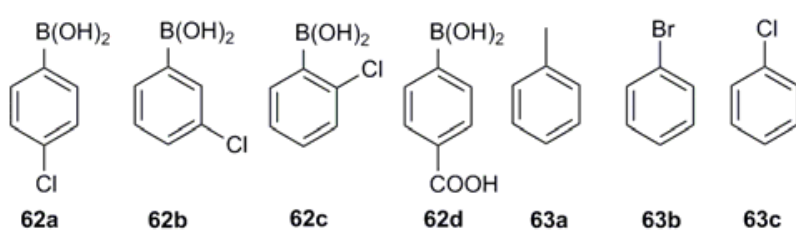

图 31 代表性的苯硼酸与芳烃 ${ }^{[39]}$ 
含氮化合物中 $\mathrm{N}$ 原子邻位 $\mathrm{C}-\mathrm{H}$ 键构建 $\mathrm{C}-\mathrm{C}$ 键的方 法 ${ }^{[37]} .2009$ 年, 涂永强课题组 ${ }^{[40]}$ 则报道了通过 $\mathrm{FeCl}_{3}$ 催化活化醇类化合物 $\mathrm{OH}$ 邻位的 $\mathrm{C}-\mathrm{H}$ 键与末端烯烃 偶联的反应，从而构建了新的 C-C 键(图 32).

研究发现二氯乙烷 (DCE) 作为反应的溶剂能够 促进该反应的发生和产物产率的提高. 如图 33 所示, 无论是伯醇 65a、65b 或者仲醇 $65 \mathrm{c}$ 在与末端烯烃 $66 \mathrm{a} 、$ 66b 或者末端烯烃的前体 66c、66d 反应时都可以得 到较好收率的偶联产物. 同时也对反应的可能机理 进行了研究, 如图 34 所示, 作者认为首先是 $\mathrm{Fe}^{\mathrm{III}}$ 催 化活化化合物醇羟基邻位的 $\mathrm{C}-\mathrm{H}$ 键, 形成中间体 $\mathbf{6 8}$, 而产生的醇类自由基再和末端烯烃进行自由基加成 形成中间体 70, 最后中间体 $\mathbf{7 0}$ 和 $[\mathrm{Fe}]-\mathrm{H}$ 经过氢转移

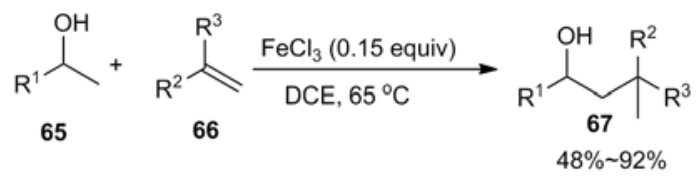

图 32 醇类化合物 $\mathrm{OH}$ 邻位的 $\mathrm{C}-\mathrm{H}$ 键活化 ${ }^{[40]}$

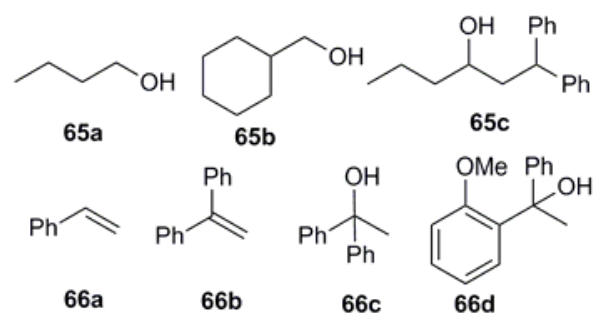

图 33 代表性的醇与烯烃底物 ${ }^{[40]}$

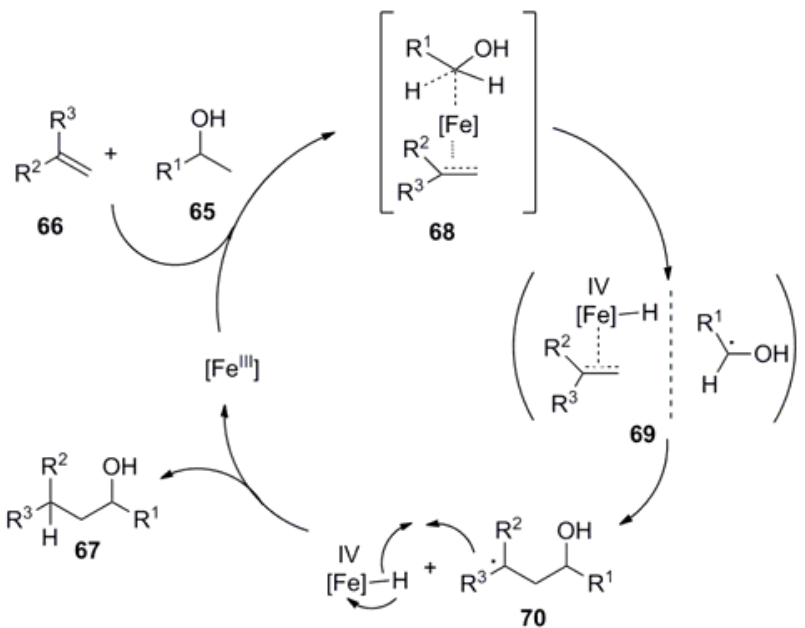

图 34 醇类化合物与末端烯烃的偶联反应机理 ${ }^{[40]}$
得到目的偶联产物, 催化剂进入下一个催化循环.

苯并杂环化合物广泛存在于香料、医药、天然产 物中 ${ }^{[41]}$, 是重要的医药和香料中间体, 关于它们的合 成方法报道的较多 ${ }^{[42]}$, 但是通过直接的 $\mathrm{C}-\mathrm{H}$ 键活化 合成的报道较少. 2009 年, 李志平课题组 ${ }^{[43]}$ 报道了 $\mathrm{FeCl}_{3}$ 催化的直接 $\mathrm{C}-\mathrm{H}$ 活化合成苯并呋喃环类化合物 的方法. 如图 35 所示, 该反应体系以 ${ }^{\mathrm{t}} \mathrm{BuOO}{ }^{\mathrm{t}} \mathrm{Bu}$ 为氧 化剂, $\mathrm{FeCl}_{3}$ 为催化剂, 苯酚 $\mathbf{7 1}$ 和二羰基化合物 $\mathbf{7 2}$ 在 DCE 中反应得到了苯并呋喃衍生物, 而且, 当体系中 有水或者醇存在时，可以促进反应的进行. 但是到目 前为止仍然不清楚水在反应中的具体作用. 研究发 现无论是苯环上还是二羰基化合物上存在的取代基 对反应的影响一般都较小，但是当苯酚类化合物的 苯环上含有强吸电子基团时，反应则无法进行.

苯并吡咯(吲哚)是另外一类重要的杂环化合物, 2009 年, Cacchi 课题组 ${ }^{[4]}$ 报道了 CuI 催化活化苯胺 类化合物 74 苯环邻位 $\mathrm{C}-\mathrm{H}$ 键合成吲哚类衍生物 $\mathbf{7 5}$ 的方法(图 36).

但是该体系却不适用于通过化合物 76 合成吲哚 衍生物. 2010 年, 梁永民课题组 ${ }^{[45]}$ 报道了 $\mathrm{FeCl}_{3}$ 催化 活化苯胺类化合物 76 苯环的 $\mathrm{C}-\mathrm{H}$ 键合成吲哚类衍生 物 77 的方法. 如图 37 所示, 在该反应中, $\mathrm{K}_{2} \mathrm{CO}_{3}$ 作为

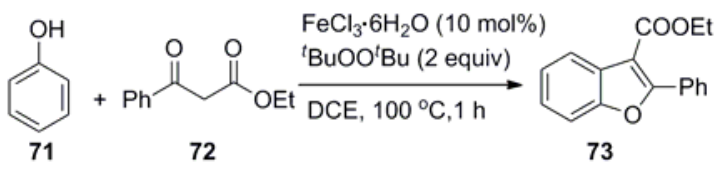

图 35 经过 C-H 键活化实现的苯并呋喃的合成 ${ }^{[43]}$

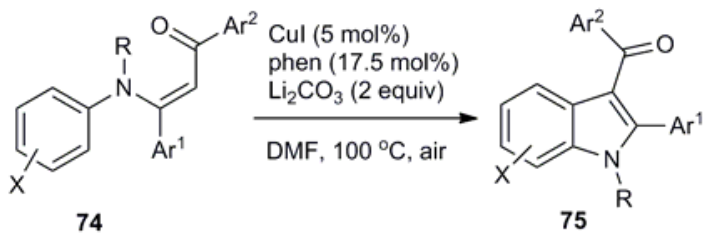

图 $36 \mathrm{CuI}$ 催化苯胺类化合物通过 $\mathrm{C}-\mathrm{H}$ 键活化的吲哚类衍 生物的合成 ${ }^{[44]}$

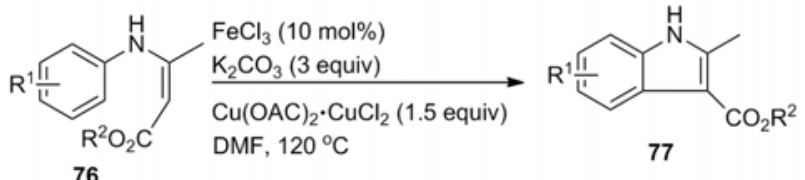

图 $37 \mathrm{FeCl}_{3}$ 催化的吲哚类衍生物的合成 ${ }^{[45]}$ 
碱, $\mathrm{Cu}(\mathrm{OAc})_{2} \cdot \mathrm{CuCl}_{2}$ 作为氧化剂, 与 $\mathrm{FeCl}_{3}$ 共同组成该 反应的催化体系.

当 $\mathrm{R}^{1}$ 在苯环 4 位时, 给电子基团比吸电子基团 对反应的影响更大. 而当 $\mathrm{R}^{1}$ 在苯环的 2 位时, 情况则 刚好相反, 而且当苯环 3,4 位为 $\mathrm{Br}$ 或者 $\mathrm{I}$ 时, 也可以 得到较好收率的环化产物, 说明该反应具有一定的 化学选择性. 该反应的缺点是对底物的要求较高, 普 适性不够广.

反应的可能机理如图 38 所示, 首先是 $[\mathrm{Fe}]$ 和 $[\mathrm{Cu}]$ 与化合物 76 形成鳌合配体 79, 然后经过电环化作用 形成中间体 80 , 最后经过质子消除和互变异构得到 吲哚衍生物 $\mathbf{7 7}$.

2010 年, 包伟良课题组 ${ }^{[46]}$ 报道了 1,3 -二芳基丙 烯类化合物 82 与芳基炔类化合物 83 的偶联反应(图 39). 该反应在温和的条件下即可发生, 但产率却较 低, 反应对底物的要求较高, 适用范围较窄. 研究表 明底物 83 苯环上有取代基时, 吸电子基团对反应的 影响大于给电子基团对反应的影响, 但是底物 82 电 子效应对反应的影响情况则恰好相反.

对于此反应, 一种可能的反应机理如图 40 所示, 首先 BQ 夺取化合物 82 中的一个质子, 形成共轭体 系中间体 86, 而中间体 85 则亲电进攻化合物 83 , 然 后和中间体 86 构建新的 $\mathrm{C}-\mathrm{C}$ 键形成偶联产物 84.

\section{4 其他价态 $\mathrm{Fe}$ 的催化}

2010 年, 李朝军课题组 ${ }^{[47]}$ 报道了利用 $\mathrm{Fe}_{3} \mathrm{O}_{4}$ 纳米

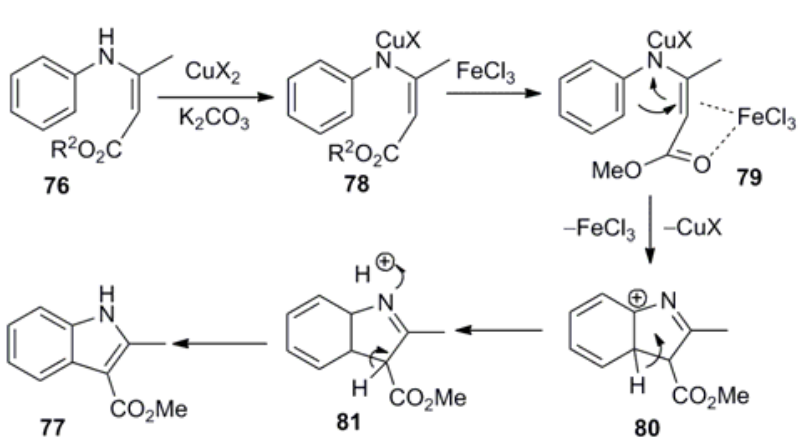

图 $38 \mathrm{FeCl}_{3}$ 催化的吲哚类衍生物的合成机理 ${ }^{[45]}$

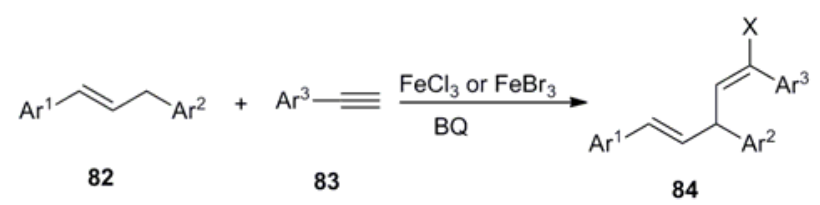

图 39 丙烯类化合物与炔的偶联 ${ }^{[46]}$

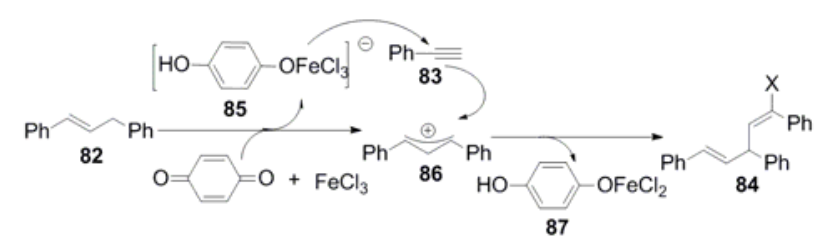

图 40 丙烯类化合物与炔的反应可能机理 ${ }^{[46]}$

粒子催化叔胺与硝基化合物的 $\mathrm{CDC}$ 反应, 实现了 $\mathrm{C}\left(\mathrm{sp}^{3}\right)-\mathrm{C}\left(\mathrm{sp}^{3}\right)$ 键的构建(图 41).

和其它 CDC 催化体系或者其他的需要氧化剂的 催化体系相比, 该体系用氧气作为氧化剂, 更加安 全, 也更符合原子经济的概念. 而且研究表明反应体 系的催化剂可以反复使用, 但催化活性并未明显降 低. 反应的副产物为水, 环保、原子利用率高, 符合 绿色化学的概念. 在该催化体系下, 丙酮亦可以和脂 肪胺发生反应, 得到类似的偶联产物(图 42).

反应机理如图 43 所示: 首先叔胺被 $\mathrm{Fe}_{3} \mathrm{O}_{4}$ 和 $\mathrm{O}_{2}$ 分子催化氧化后生成亚胺与 $\mathrm{Fe}_{3} \mathrm{O}_{4}$ 配位的中间体 $\mathbf{9 3}$,

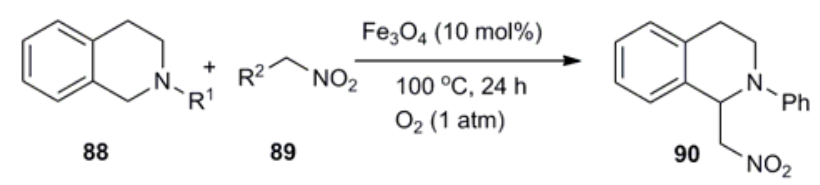

$\mathrm{R}^{1}=\mathrm{Ph}, \mathrm{PhMe}, \mathrm{PhBr}, \mathrm{PhOMe}$ $\mathrm{R}^{2}=\mathrm{H}, \mathrm{Me}, \mathrm{Et}$

图 41 叔胺与硝基化合物的 $\mathrm{CDC}$ 反应 ${ }^{[47]}$

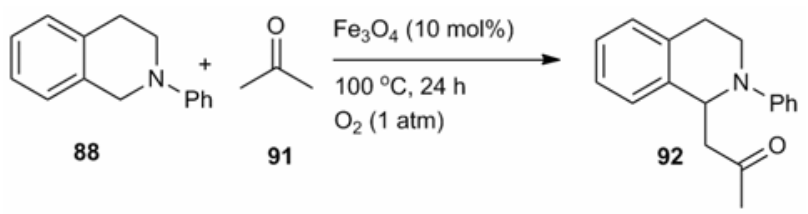

图 42 叔胺与丙酮的 $\mathrm{CDC}$ 反应 ${ }^{[47]}$

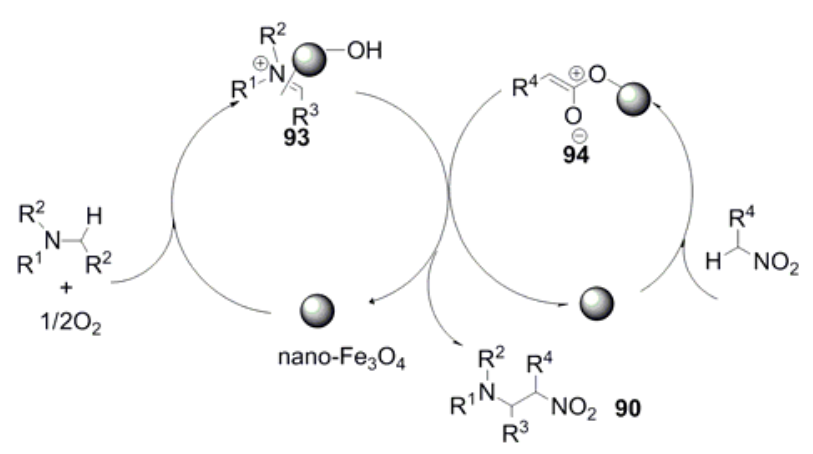

图 43 叔胺与硝基化合物的 CDC 反应的可能机理 ${ }^{[47]}$ 
$\mathrm{Fe}_{3} \mathrm{O}_{4}$ 亦可以催化硝基烷类化合物去质子化, 形成中 间体 94, 最后中间体 93 和 94 反应生成偶联产物 90 , 而且此反应机理同样适用于叔胺类化合物与丙酮的 反应.

\section{3 结论与展望}

本文总结了不同价态的 $\mathrm{Fe}$ 催化的 $\mathrm{C}-\mathrm{H}$ 键活化构 建 $\mathrm{C}-\mathrm{C}$ 键的一些最新进展, 与基于官能团化的偶联 反应相比, 通过直接的 $\mathrm{C}-\mathrm{H}$ 键活化构建 $\mathrm{C}-\mathrm{C}$ 键的反 应具有以下优点：(1) 原子利用率高, 符合原子经济 的概念. (2) 产生的副产物一般为 $\mathrm{H}_{2} 、 \mathrm{H}_{2} \mathrm{O}$ 等无毒、无 污染化合物, 符合绿色化学的概念. (3) 减少反应步
骤, 提高有机合成的效率. (4) 产物易分离, 可以减 少损失，提高产物收率. 而与其他过渡稀有贵金属相 比, 铁具有含量丰富、无毒、环境友好等优点, 是贵 金属催化剂的理想替代物.

作为近几年研究的热点, 随着人们对 $\mathrm{Fe}$ 催化的 $\mathrm{C}-\mathrm{H}$ 键活化构建 $\mathrm{C}-\mathrm{C}$ 键研究方法的不断深入, 可望 为有机合成的发展提供新的合成策略. 但是目前报 道的 $\mathrm{Fe}$ 催化的 $\mathrm{C}-\mathrm{H}$ 键构建 $\mathrm{C}-\mathrm{C}$ 键的研究中, 存在着 反应产率低、反应方法对底物的要求高、需要大量的 氧化剂等缺点, $\mathrm{Fe}$ 催化的高效高选择性的 $\mathrm{C}-\mathrm{H}$ 键活 化构建 $\mathrm{C}-\mathrm{C}$ 键的方法以及反应机理等还需要化学家 们去研究探索和发现.

\section{参考文献}

1 Lu Z, Ma SM. Metal-catalyzed enantioselective allylation in asymmetric synthesis. Angew Chem Int Ed, 2008, 47: 258-297

2 Shibasaki M, Kanai M. Asymmetric synthesis of tertiary alcohols and tertiary amines via $\mathrm{Cu}$-catalyzed C-C bond formation to ketones and ketimines. Chem Rev, 2008, 108: 2853-2873

3 Khedkar MV, Tambade PJ, Qureshi ZS, Bhanage BM. Pd/C: An efficient, heterogeneous and reusable catalyst for phosphane-free carbonylative cuzuki coupling reactions of aryl and heteroaryl iodides. Eur J Org Chem, 2010, 6981-6986

4 Kakiuchi F, Chatani N. Catalytic methods for C-H bond functionalization: Application in organic synthesis. Adv Synth Catal, 2003, 345: 1077-1101

5 Du HA, Zhang XG, Tang RY, Li JH. $\mathrm{PdCl}_{2}$-promoted electrophilic annulation of 2-alkynylphenol derivatives in the presence of iodine. $J$ Org Chem, 2009, 74: 7844-7848

6 (a) Colby DA, Bergman RG, Ellman JA. Rhodium-catalyzed C-C bond formation via heteroatom-directed C-H bond activation. Chem Rev, 2010, 110: 624-655; (b) Sun CL, Li BJ, Shi ZJ. Direct C-H transformation via iron catalysis. Chem Rev, 2011, 111: 1293-1314; (c) Ritleng V, Sirlin C, Pfeffer M. Ru-, Rh-, and Pd-catalyzed C-C bond formation involving $\mathrm{C}-\mathrm{H}$ activation and addition on unsaturated substrates: Reactions and mechanistic aspects. Chem Rev, 2002, 102: 1731-1770; (d) Corbet JP, Mignani G. Selected patented cross-coupling reaction technologies. Chem Rev, 2006, 106: 2651-2710; (e) Lyons TW, Sanford MS. Palladium-catalyzed ligand-directed C-H functionalization reactions. Chem Rev, 2010, 110: 1147-1169

7 Kleiman JP, Dubeck M. The preparation of cyclopentadienyl [ $\sigma$-phenylazo phenyl] nickel. J Am Chem Soc, 1963, 85: 1544-1545

8 (a)Martin A. Cyclometalation using d-block transition metals: Fundamental aspects and recent trends. Chem Rev, 2010, 110: 576-623; (b)Alexander DR. Mechanisms of intermolecular activation of C-H bonds in transition-metal complexs. Chem Rev, 1990, 90: 403-424

9 Zhang L, Li D, Wen TB, Herman HY, Williams ID, Lin ZY, Jia GC. Cyclometalation of 2-vinylpyridine with $\mathrm{MCl}_{2}\left(\mathrm{PPh}_{3}\right)_{3}$ and $\mathrm{MHCl}\left(\mathrm{PPh}_{3}\right)_{3}(\mathrm{M}=\mathrm{Ru}, \mathrm{Os})$. Organometallics, 2007, 26: 2849-2860

10 Lin YM, Xu H, Wen TB, He XM, Xia HP. C-H bond activation and subsequent C( $\left.\mathrm{sp}^{2}\right)$-C( $\left(\mathrm{sp}^{3}\right)$ bond formation: Coupling of bromomethyl and triphenylphosphine in an iridium complex. Organometallics, 2010, 29: 2904-2910

11 Liu B, Xie HJ, Wang HJ, Wu LQ, Zhao QY, Chen JX, Wen TB, Cao ZX, Xia HP. Selective synthesis of Osmanaphthalene and Osmanaphthalyne by intramolecular C-H activation. Angew Chem Int Ed, 2009, 48: 5461-5464

12 Arkadi V, Olivier U, Linda JWS, David YB, Martin JML, Milstein D. Formation of $\eta^{2}$ C-H agostic rhodium arene complexes and their relevance to electrophilic bond activation. J Am Chem Soc, 1998, 120: 12539-12544

13 David A, Raquel B, Marı AC, Agustı L, Rafael N, Tatiana S, Esteban PU. Regioselective ortho palladation of stabilized iminophosphoranes in Exo positions: scope, limitations, and mechanistic insights. Organometallics, 2008, 27: 2929-2936

14 Valerica P, Davit Z. New pincer-type diphosphinito (POCOP) complexes of $\mathrm{Ni}^{\mathrm{II}}$ and $\mathrm{Ni}^{\mathrm{III}}$. Chem Commun, 2007, 978-980 
15 Hans FK, Sebnem C, Robert B, Diana L, Ulrich F. Cyclometalation of substrates containing imine and pyridyl anchoring groups by iron and cobalt complexes. Angew Chem Int Ed, 2005, 44: 975-977

16 (a) Stefan A, Benjamin RE, Peter MZ, Thomas PS, Jun O. Formation of a sicationic yttrium $\eta^{2}$-pyridyl complex from an yttrium methyl dication C-H activation of pyridine. Organometallics, 2006, 25: 793-795 (b) Melanie Z, Frank E, Eberhardt H, Karl WT, Reiner A. Distinct C-H bond activation pathways in diamiao-pyridine supported rare earth metal hydrocarbyl complexs. Organometallics, 2007, 26: 6029-6041

17 (a) Burling S, Paine BM, Nama D, Brown VS, Mahon, MF, Prior TJ, Pregosin PS, Whittlesey MK, Williams JMJ. C-H activation reactions of ruthenium n-heterocyclic carbene complexes: Application in a catalytic tandem reaction involving $\mathrm{C}-\mathrm{C}$ bond formation from alcohols. $J$ Am Chem Soc, 2007, 129: 1987-1995; (b) Murahashi SI, Nakae T, Terai H, Komiya N. Ruthenium-catalyzed oxidative cyanation of tertiary amines with molecular oxygen or hydrogen peroxide and sodium cyanide: $\mathrm{sp}^{3} \mathrm{C}-\mathrm{H}$ bond activation and carbon-carbon bond formation. $J$ Am Chem Soc, 2008, 130: 11005-11012; (c) Bartoszewicz A, Martín-Matute Bn. Building molecular complexity via tandem Ru-catalyzed isomerization C-H activation. Org Lett, 2009, 11: 1749-1752; (d) Simon MO, Martinez Rm, Genet JP, Darses S. C-C bond formation via C-H bond activation under protic conditions: On the role of phosphane ligand and cosolvent. J Org Chem, 2010, 75: 208-210; (e) Yi CS, Lee DW. Intermolecular dehydrative coupling reaction of aryl ketones with cyclic alkenes catalyzed by a well-defined cationic ruthenium-hydride complex: A novel ketone olefination method via vinyl C-H bond activation. Organometallics, 2010, 29: 1883-1885

18 (a) Tsai AS, Bergman RG, Ellman JA. Asymmetric synthesis of (-)-incarvillateine employing an intramolecular alkylation via Rh-catalyzed olefinic C-H bond activation. J Am Chem Soc, 2008, 130: 6316-6317; (b) Lewis JC, Berman AM, Bergman RG, Ellman JA. Rh(I)-catalyzed arylation of heterocycles via C-H bond activation: Expanded scope through mechanistic insight. J Am Chem Soc, 2008, 130: 2493-2500; (c) Parthasarathy K, Cheng CH. Easy access to isoquinolines and tetrahydroquinolines from ketoximes and alkynes via rhodium-catalyzed $\mathrm{C}-\mathrm{H}$ bond activation. J Org Chem, 2009, 74: 9359-9364; (d) Evans ME, Li T, Jones WD. C-H vs C-C bond activation of acetonitrile and benzonitrile via oxidative addition: Rhodium vs nickel and $\mathrm{Cp}^{*}$ vs. $\mathrm{Tp}^{\prime}\left(\mathrm{Tp}^{\prime}=\right.$ hydrotris(3,5-dimethylpyrazol-1-yl)borate, $\mathrm{Cp} *=$ $\eta^{5}$-pentamethylcyclopentadienyl). J Am Chem Soc, 2010, 132: 16278-16284; (e) Ye W, Luo N, Yu Z. Rhodium-catalyzed direct alkenylation and arylation of arene $\mathrm{C}-\mathrm{H}$ bonds via decarbonylation of cinnamoyl chlorides, cinnamic anhydrides, and poly(aroyl) chlorides. Organometallics, 2010, 29: 1049-1052; (f) Hyster TK, Rovis T. Rhodium-catalyzed oxidative cycloaddition of benzamides and alkynes via C-H/N-H activation. J Am Chem Soc, 2010, 132: 10565-10569

19 (a) Lin S, Song CX, Cai GX, Wang, WH, Shi ZJ. Intra/intermolecular direct allylic alkylation via Pd(II)-catalyzed allylic C-H activation. $J$ Am Chem Soc, 2008, 130: 12901-12903; (b) Xiao Q, Wang WH, Liu G, Meng FK, Chen JH, Yang Z, Shi ZJ. Direct imidation to construct 1H-benzo[d]imidazole through Pd(II)-catalyzed C-H activation promoted by thiourea. Chem Eur J, 2009, 15: 7292-7296; (c) Reed SA, Mazzotti AR, White MC. A catalytic brønsted base strategy for intermolecular allylic C-H amination. J Am Chem Soc, 2009, 131: 11701-11706; (d) Li Y, Wang WH, Yang SD, Li BJ, Feng C, Shi ZJ. Oxidative dimerization of N-protected and free indole derivatives toward 3,3'-biindoles via Pd-catalyzed direct C-H transformations. Chem Commun, 2010, 46: 4553-4555; (e) Sun CL, Li BJ, Shi ZJ. Pd-catalyzed oxidative coupling with organometallic reagents via C-H activation. Chem Commun, 2010, 46: 677-685; (f) Wang X, Lu Y, Dai HX, Yu JQ. Pd(II)-catalyzed hydroxyl-directed C-H activation/C-O cyclization: Expedient construction of dihydrobenzofurans. $J$ Am Chem Soc, 2010, 132: 12203-12205; (g) Lu Z, Hu C, Guo J, Li J, Cui Y, Jia Y. Water-controlled regioselectivity of Pd-catalyzed domino reaction involving a C-H activation process: rapid synthesis of diverse carbo- and heterocyclic skeletons. Org Lett, 2010, 12: 480-483; (h) Michael JB, Robert JT, Richard JKT and Ian JSF. Pd-catalysed regioselective C-H functionalisation of 2-pyrones. Dalton Trans, 2010, 39 : 10391-10400; (i) Nishikata T, Abela AR, Huang S, Lipshutz BH. Cationic palladium(II) catalysis: C-H activation/suzuki-miyaura couplings at room temperature. J Am Chem Soc, 2010, 132: 4978-4979

20 Gahiez GRV, Habiak O, Gager. Efficient preparation of terminal conjugated dienes by copuling of dienol phpsphates with grignard reagents under iron catalysis. Org Lett, 2008, 10: 2382-2392

21 (a) Eiichi N, Nuoniko Y. Low valent iron-catalyzed C-C bond formation-addition, substitution, and C-H bond activation. J Org Chem, 2010, 75: 6061-6067; (b) Sun CJ, Li BJ, Shi ZJ. Direct C-H transformation via iron catalysis. Chem Rev, 2011, 111: 1293-1314

22 Jones WDJ, Glenn PF, Jeanne MP. The catalytic activation and functionalization of C-H bonds. Aldimine formation by the insertion of isonitriles into aromatic C-H bonds. J Am Chem Soc, 1987, 109: 5047-5048

$23 \mathrm{Li} \mathrm{ZP,} \mathrm{Yu} \mathrm{R,} \mathrm{Li} \mathrm{HJ.} \mathrm{Iron-catalyzed} \mathrm{C-C} \mathrm{bond} \mathrm{formation} \mathrm{by} \mathrm{direct} \mathrm{functionalization} \mathrm{of} \mathrm{C-H} \mathrm{bonds} \mathrm{adjacent} \mathrm{to} \mathrm{heteroatoms.} \mathrm{Angew} \mathrm{Chem,}$ 2008, 120: 7607-7610

24 Li HJ, He ZP, Guo XW, Li WJ, Zhao XH, Li ZP. Iron-catalyzed selective oxidation of $N$-methyl amines: Highly efficient synthesis of methylene-bridged bis-1,3-dicarbonyl compounds. Org Lett, 2009, 11: 4176-4179

$25 \mathrm{Li} \mathrm{Z}$, Cao L, Li CJ. $\mathrm{FeCl}_{2}$-catalyzed selective $\mathrm{C}-\mathrm{C}$ bond formation by oxidative activation of a benzylic C-H bond. Angew Chem Int Ed, 
2007, 46: 6505-6507

26 Li ZP, Li CJ. Highly efficient copper-catalyzed nitro-mannich type reaction: Cross-dehydrogenative-coupling between $\mathrm{sp}^{3} \mathrm{C}-\mathrm{H}$ bond and sp ${ }^{3}$ C-H bond. J Am Chem Soc, 2005, 127: 3672-3673

27 Zhang Y, Li CJ. Highly efficient direct alkylation of activated methylene by cycloalkanes. Eur J Org Chem, 2007, 4654-4657

$28 \mathrm{Li}$ YZ, Li BJ, Lu XY, Lin S, Shi ZJ. Cross dehydrogenative arylation (CDA) of a benzylic C-H bond with arenes by iron catalysis. Angew Chem Int Ed, 2009, 48: 3817-3820

29 Song CX, Cai G.X, Farrell TR., Jiang ZP, Li H, Gan LB, Shi ZJ. Direct functionalization of benzylic C-Hs with vinyl acetates via Fe-catalysis. Chem Commun, 2009, 6002-6004

30 (a) Proch S, Kempe R. An efficient bimetallic rhodium catalyst for the direct arylation of unactivated arenes. Angew Chem Int Ed, 2007, 46: 3135-3138; (b) Lafrance M, Fagnou K. Palladium-catalyzed benzene arylation: Incorporation of catalytic pivalic acid as a proton shuttle and a key element in catalyst design. J Am Chem Soc, 2006, 128: 16496-16497; (c) Ackermann L, Novak P, Vicente R, Hofmann N. Ruthenium-catalyzed regioselective direct alkylation of arenes with unactivated alkyl halides through $\mathrm{C}-\mathrm{H}$ bond cleavage. Angew Chem Int Ed, 2009, 48: 6045-6048; (d) Tobisu M, Hyodo I, Chatani N. Nickel-catalyzed reaction of arylzinc reagents with N-aromatic heterocycles: A straightforward approach to $\mathrm{C}-\mathrm{H}$ bond arylation of electron-deficient heteroaromatic compounds. J Am Chem Soc, 2009, 131: 12070-12071

31 Vallée Fdr, Mousseau JJ, Charette AB. Iron-catalyzed direct arylation through an aryl radical transfer pathway. J Am Chem Soc, 2010, 132: $1514-1516$

32 Volla CMR, Vogel P. Chemoselective $\mathrm{C}-\mathrm{H}$ bond activation: Ligand and solvent free iron-catalyzed oxidative C-C cross-coupling of tertiary amines with terminal alkynes. Reaction scope and mechanism. Org Lett, 2009, 11: 1701-1704

33 Norinder J, Matsumoto A, Yoshikai N, Nakamura E. Iron-catalyzed direct arylation through directed C-H bond activation. J Am Chem Soc, 2008, 130: 5858-5859

34 Ilies L, Tsuji H, Nakamura E. Synthesis of benzo[b]siloles via KH-promoted cyclization of (2-alkynylphenyl) silanes. Org Lett, 2009, 11: 3966-3968

35 Nakamura E, Yoshikai N, Matsumoto A, Norinder J. Iron-catalyzed direct arylation of aryl pyridines and imines using oxygen as an oxidant. Synlett, 2010, 313-316

36 Yoshikai N, Matsumoto A, Norinder J, Nakamura E. Iron-catalyzed chemoselective ortho arylation of aryl imines by directed C-H bond activation. Angew Chem Int Ed, 2009, 48: 2925-2928

37 Yoshikai N, Mieczkowski A, Matsumoto A, Ilies L, Nakamura E. Iron-catalyzed C-C bond formation at $\alpha$-position of aliphatic amines via C-H bond activation through 1,5-hydrogen transfer. J Am Chem Soc, 2010, 132: 5568-5569

38 Yin LX, Jurgen L. Carbon-corbon coupling reactions catalyzed by heterogeneous palladium catalysts. Chem Rev, 2007, 107: 133-173

39 Wen J, Zhang J, Chen SY, Li J, Yu XQ. Iron-mediated direct arylation of unactivated arenes. Angew Chem, 2008, 120: 9029-9032

40 Zhang SY, Tu YQ, Fan CA, Zhang FM, Shi L. Iron-catalyzed $\mathrm{C}\left(\mathrm{sp}^{3}\right)-\mathrm{C}\left(\mathrm{sp}^{3}\right)$ bond formation through $\mathrm{C}\left(\mathrm{sp}^{3}\right)-\mathrm{H}$ functionalization: a cross-coupling reaction of alcohols with alkenes. Angew Chem Int Ed, 2009, 48: 8761-8765

41 (a) Yang J, Wu H, Shen L, Qin Y. Total synthesis of ( \pm )-communesin F. J Am Chem Soc, 2007, 129: 13794-13795; (b) Herzon SB, Myers AG. Enantioselective synthesis of stephacidin B. J Am Chem Soc, 2005, 127: 5342-5344; (c) Baran PS, Guerrero CA, Hafensteiner BD, Ambhaikar NB. Total synthesis of avrainvillamide (CJ-17,665) and stephacidin B. Angew Chem Int Ed, 2005, 44: 3892-3895

42 (a) Humphrey GR, Kuethe JT. Practical methodologies for the synthesis of indoles. Chem Rev, 2006, 106: 2875-2911; (b) Patil NT, Yamamoto Y. Coinage metal-assisted synthesis of heterocycles. Chem Rev, 2008, 108: 3395-3442; (c) Zeni G, Larock RC. Synthesis of heterocycles via palladium-catalyzed oxidative addition. Chem Rev, 2006, 106: 4644-4680; (d) Cacchi S, Fabrizi G. Synthesis and functionalization of indoles through palladium-catalyzed reactions. Chem Rev, 2005, 105: 2873-2920; (e) Alonso F, Beletskaya IP, Yus M. Transition-metal-catalyzed addition of heteroatom-hydrogen bonds to alkynes. Chem Rev, 2004, 104: 3079-3160; (f) Horton DA, Bourne GT, Smythe ML. The combinatorial synthesis of bicyclic privileged structures or privileged substructures. Chem Rev, 2003, 103: 893-930

43 Guo XW, Yu R, Li HJ, Li ZP. Iron-catalyzed tandem oxidative coupling and annulation: An efficient approach to construct polysubstituted benzofurans. J Am Chem Soc, 2009, 131: 17387-17393

44 Bernini R, Fabrizi G, Sferrazza A, Cacchi S. Copper-catalyzed C-C bond formation through C-H functionalization: Synthesis of multisubstituted indoles from $N$-aryl enaminones. Angew Chem Int Ed, 2009, 48: 8078-8081

45 Guan ZH, Yan ZY, Ren ZH, Liu XY, Liang YM. Preparation of indoles via iron catalyzed direct oxidative coupling. Chem Commun, 2010, 46: $2823-2825$

46 Mo H, Bao WL, Iron-promoted synthesis of substituted 1-halo-1,4-pentadienes by reaction of 1,3-diarylpropenes with ethynylbenzenes via 
$\mathrm{sp}^{3} \mathrm{C}-\mathrm{H}$ bond activation. J Org Chem, 2010, 75: 4856-4859

47 Li CJ, Song G, Zeng T, Moores A. Magnetically recoverable iron nanoparticle catalyzed cross-dehydrogenative coupling (CDC) between two C(sp $\left.{ }^{3}\right)-H$ bonds using molecular oxygen. Synlett 2010, 2002-2008

\title{
Iron catalyzed $\mathrm{C}-\mathrm{C}$ bond formation via $\mathrm{C}-\mathrm{H}$ activation
}

\author{
NIU Po \& WEN TingBin \\ Departemt of Chemistry, College of Chemistry and Chemical Engineering, Xiamen University, Xiamen 361005, China
}

\begin{abstract}
Transition metal-catalyzed $\mathrm{C}-\mathrm{H}$ bond activation followed by $\mathrm{C}-\mathrm{C}$ bond formation has attracted great attention due to its high synthetic efficiency and atom economy. Iron as one of the most abundant metals on earth, which offers several advantages such as inexpensive, readily available and environmentally friendly, has been increasingly explored in modern catalysis. In particular, recent studies on Fe catalysis have led to much progress in the direct formation of $\mathrm{C}-\mathrm{C}$ bonds through iron-catalyzed $\mathrm{C}-\mathrm{H}$ bond activation. In this paper, the recent advances in iron-catalyzed the $\mathrm{C}-\mathrm{C}$ bond formation via $\mathrm{C}-\mathrm{H}$ bond activation were summarized and categorized according to the oxidation states of the iron catalysts. The mechanisms of these transformations were also included.
\end{abstract}

Keywords: iron, catalysis, $\mathrm{C}-\mathrm{H}$ bond activation, $\mathrm{C}-\mathrm{C}$ bond formation 